\title{
Planet Occurrence: Doppler and Transit Surveys
}

Joshua N. Winn

\begin{abstract}
Prior to the 1990s, speculations about the occurrence of planets around other stars were based only on planet formation theory, observations of circumstellar disks, and the knowledge that at least one seemingly ordinary star had managed to make a variety of different planets. Since then, Doppler and transit surveys have revealed the population of planets around other Sun-like stars, especially those with orbital periods shorter than a few years. Over the last decade these surveys have risen to new heights with Doppler spectrographs capable of $1 \mathrm{~m} \mathrm{~s}^{-1}$ precision, and space telescopes capable of detecting the transits of Earth-sized planets. This article is a brief introductory review of the knowledge of planet occurrence that has been gained from these surveys.
\end{abstract}

\section{Introduction}

If, in some cataclysm, all our knowledge of exoplanets were to be destroyed, and only one sentence passed on to the next generation of astronomers, what statement would contain the most helpful information? 1 Here is one possibility: Most Sun-like stars have planets, which display a wider range of properties - size, mass, orbital parameters - than the planets of the Solar System.

To be quantitative we could give the fraction of stars with planets, restricted to the types of planets we have managed to detect. But since this fraction is so close to unity, it might be more helpful to specify the average number $n$ of planets per star: the total number of planets in the galaxy divided by the number of stars. Better still would be a mathematical function to show how occurrence depends on the planet's properties. For example, we could supply a functional form for

Department of Astrophysical Sciences, Princeton University, 4 Ivy Lane, Princeton, NJ 08544, USA, e-mail: jnwinneprinceton.edu

${ }^{1}$ Adapted from book I, chapter 1, verse 2 of Feynman (1963). 


$$
\Gamma_{R, P}=\frac{\partial^{2} n}{\partial \log R \partial \log P},
$$

the average number of planets per star per log-intervals in radius and period. The number $n$ is the occurrence rate and the function $\Gamma_{R, P}$ is an occurrence rate density.

Occurrence rates depend on other planetary parameters, such as orbital eccentricity, and on the characteristics of the star, such as mass and metallicity. Occurrence is also conditional on the properties of any other planets known to exist around the same star. No simple function could account for all these parameters and their correlations. Ideally, we would transmit a computer program that produces random realizations of planetary systems that are statistically consistent with everything we have learned from planet surveys. This would help our descendants design new instruments to detect planets, and inspire their theories for planet formation.

What follows is an introductory review of the progress toward this goal that has come from Doppler and transit surveys. The basics of the Doppler and transit methods themselves are left for other reviews, such as those by Lovis and Fischer (2010), Winn (2010), and Wright (this volume). Here we will simply remind ourselves of the key properties of the Doppler and transit signals:

$$
\begin{aligned}
& K=\frac{0.64 \mathrm{~m} \mathrm{~s}^{-1}}{\sqrt{1-e^{2}}}\left(\frac{P}{1 \text { day }}\right)^{-1 / 3} \frac{\left(M / M_{\oplus}\right) \sin I}{\left(M_{\star} / M_{\odot}\right)^{2 / 3}}, \\
& \delta=8.4 \times 10^{-5}\left(\frac{R / R_{\star}}{R_{\oplus} / R_{\odot}}\right)^{2}, p_{\text {tra }}=\frac{0.0046}{1-e^{2}} \frac{R_{\star} / a}{R_{\odot} / 1 \mathrm{AU}}
\end{aligned}
$$

where $K$ is the radial-velocity semiamplitude; $\delta$ is the fractional loss of light during transits; $p_{\text {tra }}$ is the probability for a randomly-oriented orbit to exhibit transits; $a, P$, $e$, and $I$ are the orbital semimajor axis, period, eccentricity, and inclination; $M$ and $R$ are the mass and radius of the planet, and $M_{\star}$ and $R_{\star}$ are those of the star.

The next section describes methods for occurrence calculations. Because the surveys have shown major differences in occurrence between giant planets and small planets, with a dividing line just above $4 R_{\oplus}$ or $20 M_{\oplus}$, the results for giants and small planets are presented separately. After that comes a review of what is known about other types of stars, followed by a discussion of future prospects.

\section{Methods}

Life would be simple if planets came in only one type and we could detect them unerringly. We would search $N$ stars, detect $N_{\text {det }}$ planets, and conclude $n \approx N_{\text {det }} / N$. But detection is not assured: small signals can be lost in the noise. If the detection probability were $p_{\operatorname{det}}$ in all cases, then effectively we would only have searched $p_{\text {det }} N$ stars, and the estimated occurrence rate would be $N_{\text {det }} /\left(p_{\text {det }} N\right)$.

In reality, $p_{\text {det }}$ depends strongly on the characteristics of the star and planet (see Figures 1 and 22. Detection is easier for brighter stars, shorter orbital periods, and 


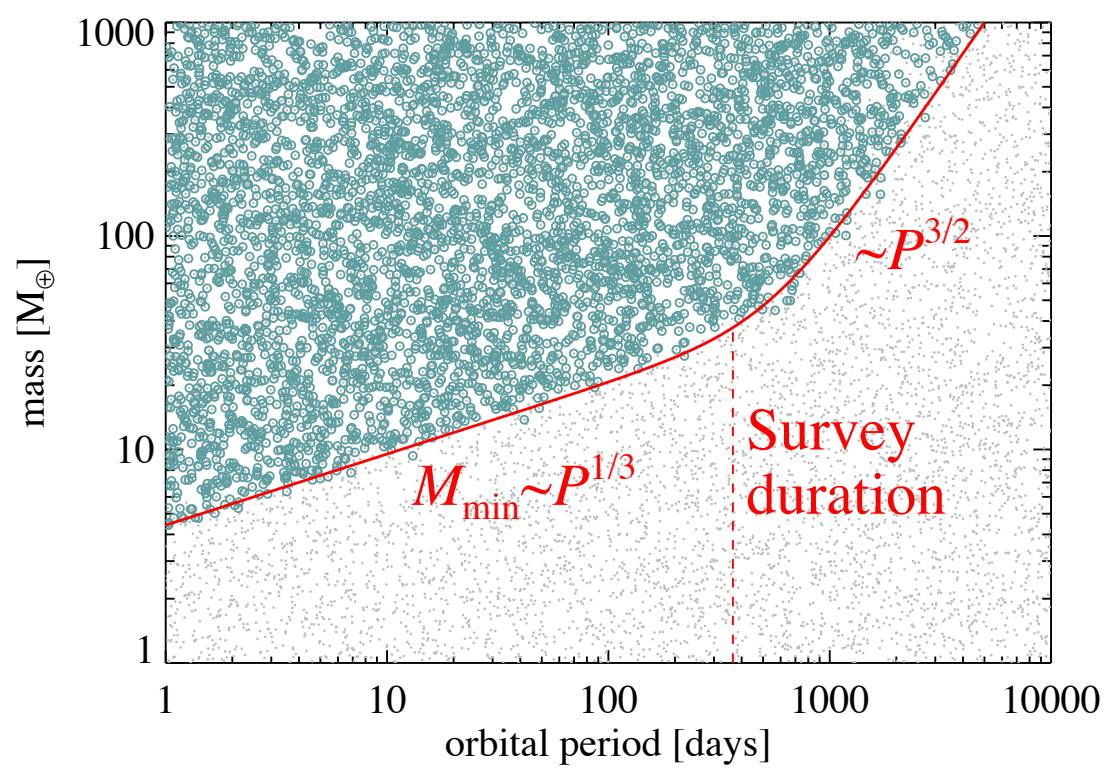

Fig. 1 Idealized Doppler survey of $10^{4}$ identical Sun-like stars. Each star has one planet on a randomly-oriented circular orbit, with a mass and period drawn from log-uniform distributions between the plotted limits. Each star is observed 50 times over one year with $1 \mathrm{~m} \mathrm{~s}^{-1}$ precision. The small gray dots are all the planets; the blue circles enclose those detected with $10 \sigma$ confidence. For periods shorter than the survey duration, the threshold mass is proportional to $P^{1 / 3}$, corresponding to a constant Doppler amplitude. For longer periods, the threshold mass increases more rapidly, with an exponent depending on the desired false-alarm probability (Cumming 2004).

larger planets relative to the star. For this reason we need to group the planets according to orbital period and other salient characteristics for detection: the radius $R$, for transit surveys; and $m \equiv M \sin I$ for Doppler surveys. Then our estimate becomes

$$
n_{i} \approx \frac{N_{\mathrm{det}, i}}{\sum_{j=1}^{N} p_{\mathrm{det}, i j}}
$$

where the index $i$ refers to a group of planets sharing the same characteristics, and the index $j$ specifies the star that was searched. Transit surveys have the additional problem that most planets produce no signal at all, because their orbits are not viewed at high enough inclination. Thus we must also divide by the probability $p_{\text {tra }}$ for transits to occur.

This conceptually simple method has been the basis of many investigations. The results of Doppler surveys are presented as a matrix of occurrence rates for rectangular regions in the space of $\log m$ and $\log P$; for transit surveys the regions are in the space of $\log R$ and $\log P$. Ideally, each region is large enough to contain many 


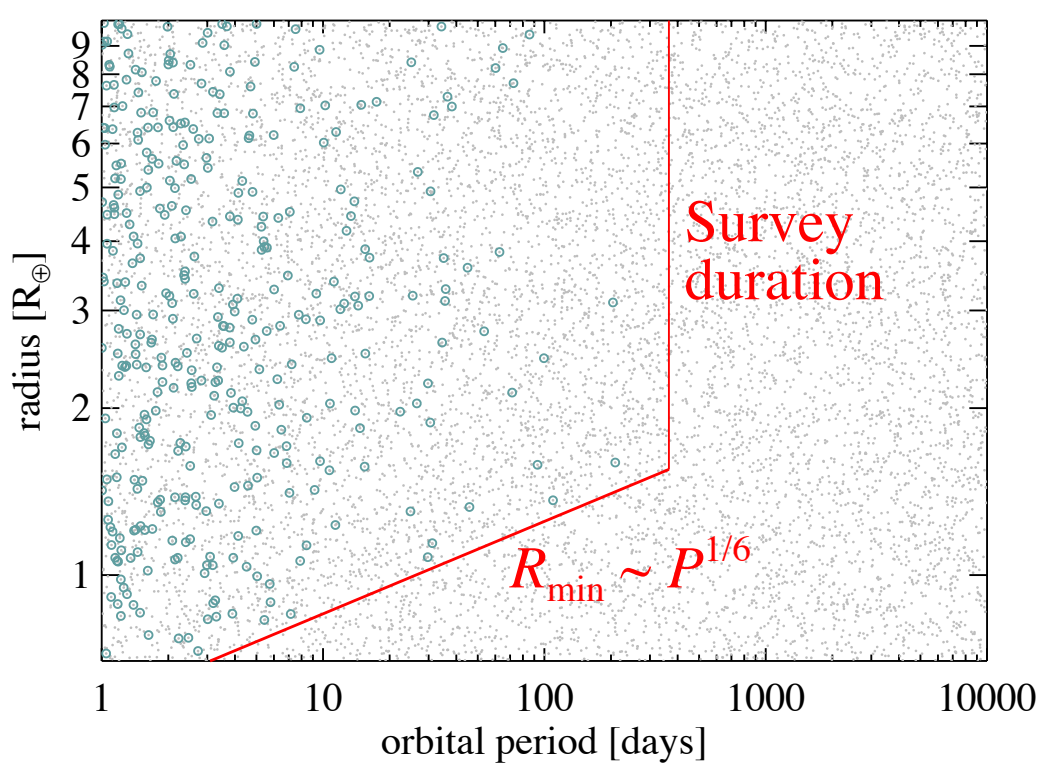

Fig. 2 Idealized transit survey of $10^{4}$ identical Sun-like stars. Each star has one planet on a randomly-oriented circular orbit, with a radius and period drawn from log-uniform distributions between the plotted limits. Each star is observed continuously for one year with a photon-limited photometric precision corresponding to $3 \times 10^{-5}$ over 6 hours. The small gray dots are all the planets; the blue circles enclose those detected with $10 \sigma$ confidence based on at least two transit detections. Compared to the Doppler survey, the transit survey finds fewer planets and is more strongly biased toward short periods, because of the geometric transit probability is low and proportional to $P^{-2 / 3}$. For orbital periods shorter than survey duration, the threshold radius varies as $P^{1 / 6}$ (Pepper et al. 2003). For longer periods it is impossible to observe more than one transit.

detected planets, and yet small enough that the detection probability does not vary too much from one side to the other.

In practice these conditions are rarely achieved, and other methods are preferred. One approach is to posit a parameterized functional form for the occurrence rate density, such as a power law

$$
\Gamma_{m, P}=\frac{\partial^{2} n}{\partial \log m \partial \log P}=C m^{\alpha} P^{\beta},
$$

and use it to construct a likelihood function for the outcome of a survey. This function must take into account the detection probability, the properties of the detected systems, and the properties of the stars for which no planets were detected. Then the values of the adjustable parameters are determined by maximizing the likelihood. Details are provided by Tabachnik and Tremaine (2002), Cumming et al. (2008), and Youdin (2011). Foreman-Mackey et al. (2014) cast the problem in the form 
of Bayesian hierarchical inference, emphasizing the importance of accounting for observational uncertainties in the planet and stellar properties.

Most studies report the occurrence rate density as a function of planet properties, regardless of any other planets in the system. It is more difficult to quantify the multiplicity of planetary systems, the number of planets that orbit together around the same star. For Doppler surveys, one problem is that the star is pulled by all the planets simultaneously. As a result, the detectability of a given planet depends on the properties of any other planets - especially their periods - and on the timespan and spacing between the data points. This makes it difficult to calculate the detection probabilities. For transit surveys, the overlap between different planetary signals is minimal; instead the problem is a degeneracy between multiplicity and inclination dispersion. A star with only one detected planet could lack additional planets, or it could have several planets only one of which happens to transit. In principle this degeneracy can be broken by combining the results of Doppler and transit surveys (Tremaine and Dong|2012).

Doppler surveys have uncovered a total of about 500 planets. The most informative surveys for planet occurrence were based on observations with the High Resolution Echelle Spectrometer (HIRES) on the Keck I 10-meter telescope (Cumming et al. 2008; Howard et al. 2010) and the High Accuracy Radial-velocity Planet Searcher (HARPS) on the La Silla 3.6-meter telescope (Mayor et al. 2011). Both instruments were used to monitor $\sim 10^{3}$ stars for about a decade, with a precision of a few meters per second. Additional information comes from a few lower-precision and longer-duration surveys (see, e.g., Lovis and Fischer 2010).

For transits, the ground-based surveys have discovered about 200 planets, but are not well suited to occurrence calculations because the sample of searched stars and the detection probabilities are poorly characterized. Instead our most important source is the NASA Kepler mission, which used a 1-meter space telescope to measure the brightness of 150,000 stars every 30 minutes for 4 years (Borucki 2016). The typical photometric precision over a 6-hour time interval was of order $10^{-4}$. This was sufficient to detect several thousand planets.

\section{Giant planets}

Overall occurrence For giant planets the key references are Cumming et al. (2008) and Mayor et al. (2011) for Doppler surveys, and Santerne et al. (2016) for Kepler. These studies agree that giant planets with periods shorter than a few years are found around $\approx 10 \%$ of Sun-like stars (see Figure 3 . In particular Cumming et al. (2008) studied planets with a minimum mass $m$ in the range from $0.3-10 M_{\mathrm{Jup}}$ and $P$ from 2-2000 days. They fitted a power-law of the form given by Equation 5 , finding $\alpha=-0.31 \pm 0.20$ and $\beta=0.26 \pm 0.10$, normalized such that $10.5 \%$ of Sun-like 
stars have such a planet 2 They found the data to be equally well described by a distribution uniform in $\log P$ from 2-300 days (i.e., $\beta=0$ ), followed by a sharp increase by a factor of 4-5 for longer periods. The Kepler data are also consistent with the latter description (Santerne et al. 2016). This uptick in planet occurrence at long periods might be related to the location of the "snow line" in protoplanetary disks, which plays a role in the theory of giant-planet formation via core accretion; beyond this line there is enough snow (frozen volatiles) to pack onto a growing protoplanet and help it to achieve the critical mass for runaway accretion of hydrogen and helium gas (Pollack et al. 1996; , Lecar et al. 2006).

Metallicity The earliest Doppler surveys revealed the occurrence of giant planets with periods shorter than a few years to be a steeply rising function of the host star's metallicity (Santos et al. 2003, Fischer and Valenti 2005). This too is widely interpreted as support for core accretion theory. The logic is that the rapid assembly of a massive solid core - an essential step in the theory - is easier to arrange in a metal-rich protoplanetary disk. Fischer and Valenti (2005) found their sample of Doppler-detected giant planets to be compatible with $n \propto z^{2}$, where $z$ is the iron-tohydrogen abundance relative to the solar value. Most recently, Petigura et al. (2018) used Kepler data to determine the best-fitting parameters of

$$
\Gamma_{P, z}=\frac{\partial^{2} N}{\partial \log P \partial \log z}=C P^{\alpha} z^{\beta}
$$

For hot Jupiters they found $\beta=3.4 \pm 0.9$, a remarkably strong dependence. However, for companions more massive than $4 M_{\text {Jup }}$, Santos et al. (2017) found the association with high metallicity to be much weaker or absent, suggesting that such objects do not form through core accretion. Schlaufman (2018) reached the same conclusion with a sample spanning a larger range of companion masses, and went so far as to say that companions more massive than $10 M_{\text {Jup }}$ should not be considered planets. The metallicity effect is also weaker for planets smaller than Neptune (Buchhave et al. 2012), although for orbital periods shorter than about 10 days, even small planets are associated with elevated metallicity (Mulders et al. 2016; Wilson et al. 2018; Petigura et al. 2018).

Hot Jupiters Easy to detect, but intrinsically uncommon, hot Jupiters have an occurrence rate of $0.5-1 \%$ for periods between 1 and 10 days. They are even rarer for periods shorter than one day (Howard et al. 2012, Sanchis-Ojeda et al. 2014). There is a $\approx 2 \sigma$ discrepancy between the rate of $0.8-1.2 \%$ measured in Doppler surveys (Wright et al. 2012; Mayor et al. 2011) and 0.6\% measured using Kepler data (Howard et al.2012, Petigura et al.2018). This is despite the similar metallicity distributions of the stars that were searched (Guo et al.2017). While we should never lose too much sleep over $2 \sigma$ discrepancies, it might be caused by misclassified stars and unresolved binaries in the Kepler sample (Wang et al.|2015).

\footnotetext{
2 Technically, Cumming et al. 2008) calculated the fraction of stars with planets, rather than the average number of planets per star. The value of $C$ is $1.04 \times 10^{-3}$ when mass is measured in $M_{\text {Jup }}$ and period is measured in days.
} 
Jupiter analogs A perennial question is whether the Solar System is typical or unusual in some sense. It is difficult to answer because the current Doppler and transit surveys are only barely sensitive to the types of planets found in the Solar System: the inner planets are too small and the outer planets have periods that are too long. The most easily detected planet in an extraterrestrial Doppler survey would probably be Jupiter; hence, a few groups have tried to quantify the occurrence of solar-like systems by searching for Jupiter-like exoplanets. Wittenmyer et al. (2016) presented the latest effort, finding the occurrence rate to be $6.2_{-1.6}^{+2.8} \%$ for planets of mass 0.3-13 $M_{\text {Jup }}$ with orbital distances from 3-7 AU and eccentricities smaller than 0.3. Of course the rate depends on the definition of "Jupiter analog", a term without a precise meaning. The same problem arises when trying to measure the occurrence of "Earth-like" planets.

Long-period giants Regarding the more general topic of wide-orbiting giant planets, Foreman-Mackey et al. (2016) measured the occurrence of "cold Jupiters" with periods ranging from 2-25 years, by searching the Kepler data for stars showing only one or two transits over 4 years. For planets with $R=0.4-1.0 R_{\text {Jup }}$ they found

$$
\Gamma_{R, P}=\frac{\partial^{2} n}{\partial \log R \partial \log P}=0.18 \pm 0.07 \text {. }
$$

Integrating over the specified ranges of radius and period gives a total occurrence rate of $0.42 \pm 0.16$ planets per star.

Bryan et al. (2016) studied the occurrence of long-period giants conditioned on the detection of a shorter-period giant. Using high-resolution imaging and long-term Doppler monitoring, they searched for wide-orbiting companions to 123 giant planets with orbital distances ranging from 0.01 to $5 \mathrm{AU}$. They found the occurrence of outer companions to be higher than would be predicted by extrapolating the powerlaw of Cumming et al. (2008) to longer periods. They also found $d n / d \log P$ to decline with period, unlike the more uniform distribution observed for closer-orbiting giant planets. The occurrence rate was $(53 \pm 5) \%$ for outer companions of mass 1-20 $M_{\text {Jup }}$ and orbital distance 5-20 AU.

Other properties The giant-planet population is distinguished by other features. Their orbits show a broad range of eccentricities (see, e.g., Udry and Santos 2007). Their occurrence seems to fall precipitously for masses above $\approx 10 M_{\text {Jup }}$, at least for orbital distances shorter than a few AU. Because of this low occurrence, the mass range from 10-80 $M_{\text {Jup }}$ is often called the "brown dwarf desert" (Grether and Lineweaver 2006; Sahlmann et al.|2011; Triaud et al. 2017). As mentioned earlier, the inhabitants of this desert are not strongly associated with high-metallicity stars, unlike Jovian-mass planets(Santos et al. 2017; Schlaufman 2018). Occasionally we find two giant planets in a mean-motion resonance (Wright et al. 2011). The rotation of the star can be grossly misaligned with the orbit of the planet, especially if the star is more massive than about 1.2 $M_{\odot}$ (Triaud, this volume). These and other topics were reviewed recently by Winn and Fabrycky (2015) and Santerne (this volume). 


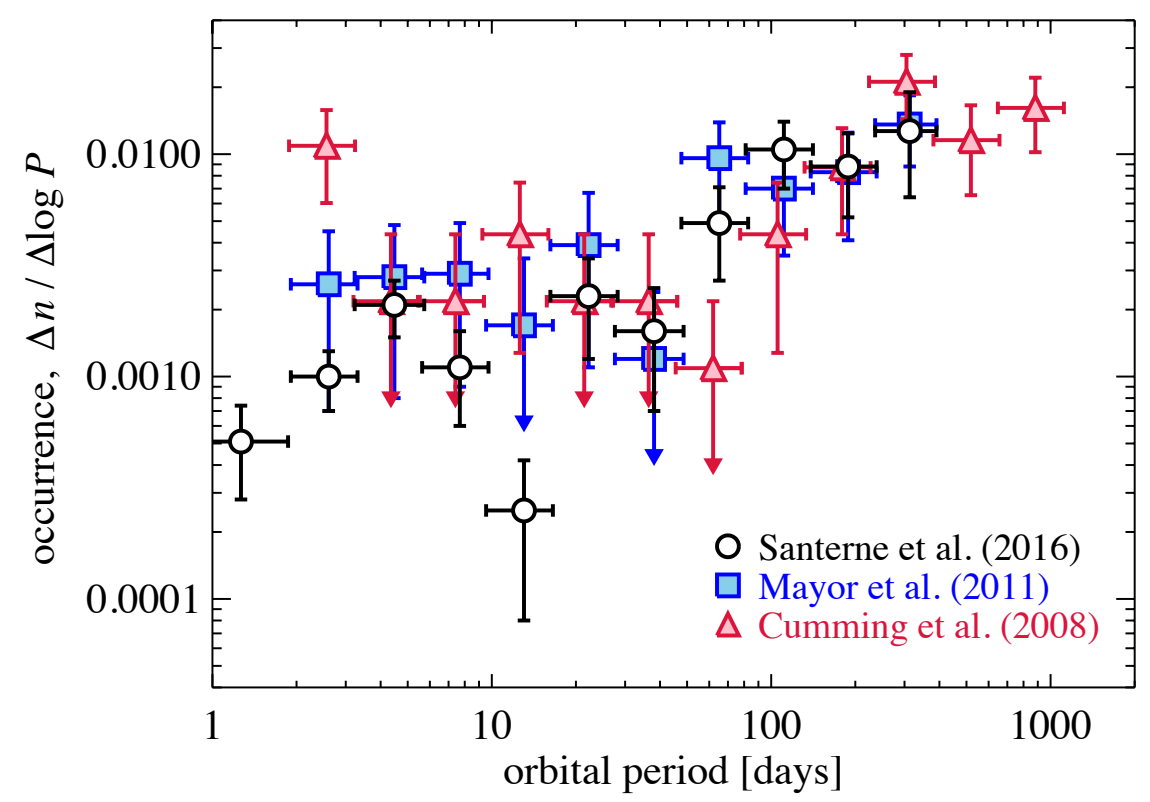

Fig. 3 Occurrence of giant planets as a function of orbital period, from the Kepler transit survey and two independent Doppler surveys. Data from Cumming et al. (2008) refer to planets with minimum mass $m>0.3 M_{\text {Jup }}$; data from Mayor et al. (2011) are for planets with $m>0.16 M_{\text {Jup }}$; data from (Santerne et al. 2016) are for planets with a radius in the approximate range $0.5-2 R_{\text {Jup }}$. Rates are reported in planets per star per $\Delta \log P=0.23$ (the range indicated by the horizontal error bars). Downward-pointing arrows indicate upper limits.

\section{Smaller planets}

Overall occurrence About half of Sun-like stars have at least one planet with an orbital period shorter than 100 days, and a size in between those of Earth and Neptune. Planet formation theories generally did not predict this profusion of close-orbiting planets. Indeed some of the most detailed theories predicted that close-in "superEarth" or "sub-Neptune" planets would be especially rare (Ida and Lin 2008). Their surprisingly high abundance led to new theories in which small planets can form in short-period orbits, rather than forming farther away from the star and then migrating inward (see, e.g., Hansen and Murray|2012, Chiang and Laughlin|2013).

Doppler surveys provided our first glimpse at this population of planets, and then Kepler revealed it in vivid detail. For planets with periods shorter than 50 days and minimum masses between 3 and $30 M_{\oplus}$, two independent Doppler surveys found the occurrence rate to be $(15 \pm 5) \%$ (Howard et al. 2010) and $(27 \pm 5) \%$ (Mayor et al. 2011). For this same period range, and planets with a radius between 2 and $4 R_{\oplus}$, analysis of Kepler data gave an occurrence rate of $(13.0 \pm 0.8) \%$ (Howard 
et al. 2012). The results of these surveys are compatible, given reasonable guesses for the relation between planetary mass and radius (Howard et al. 2012; Figueira et al. 2012; Wolfgang and Laughlin|2012).

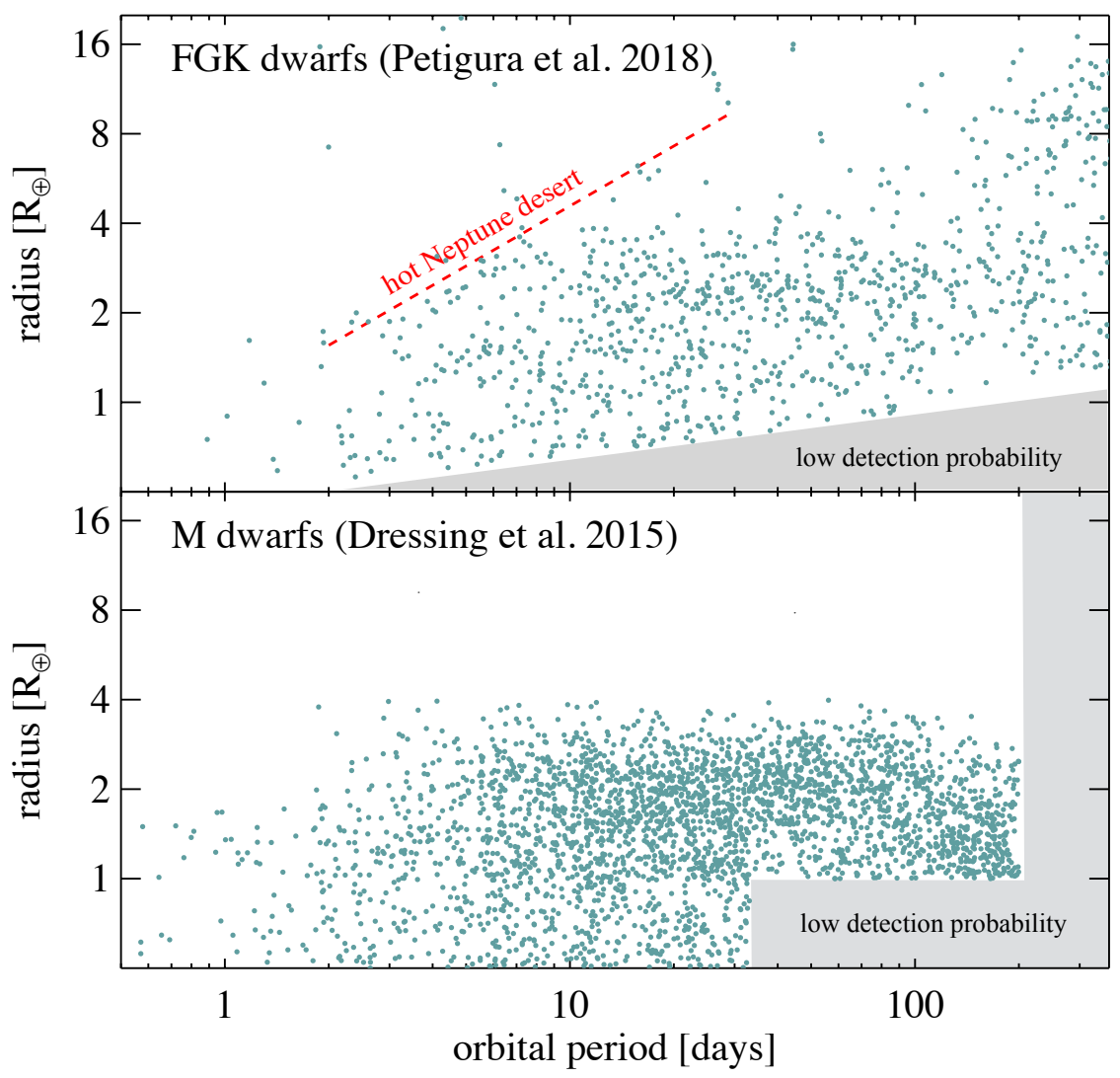

Fig. 4 Planet occurrence around FGK dwarfs (top) and M dwarfs (bottom) based on Kepler data. The blue dots represent a random sample of planets around $10^{3}$ stars, drawn from the occurrence rate densities of Petigura et al. (2018) and Dressing and Charbonneau 2015). Compared to FGK stars, the $\mathrm{M}$ stars have a higher occurrence of small planets and a lower occurrence of giant planets. For the $\mathrm{M}$ dwarfs, occurrence rates for planets larger than $4 R_{\oplus}$ were not reported because only four planet candidates in that range were detected.

Size, mass, and period The surveys also agree that within this range of periods and planet sizes, the occurrence rate is higher for the smallest planets, roughly according to power laws (Howard et al. 2010, 2012):

$$
\frac{d n}{d \log m} \propto m^{-0.5}, \frac{d n}{d \log R} \propto R^{-2} .
$$


For even smaller or longer-period planets, Kepler provides almost all the available information. Figure 4 shows some of the latest results (see also Fressin et al.2013, Burke et al. 2015). The period distribution $d n / d \log P$ rises as $\approx P^{2}$ between 1-10 days, before leveling off to a nearly constant value between 10-300 days.

Multiple-planet systems Small planets occur frequently in closely-spaced systems (Mayor et al.2011, Lissauer et al. 2011), with as many as eight planets with periods shorter than a year (Shallue and Vanderburg 2018). The period ratios tend to be in the neighborhood of 1.5-5 (Fabrycky et al. 2014). In units of the mutual Hill radius,

$$
a_{\mathrm{H}} \equiv\left(\frac{M_{\mathrm{in}}+M_{\mathrm{out}}}{3 M_{\star}}\right)^{1 / 3}\left(\frac{a_{\mathrm{in}}+a_{\mathrm{out}}}{2}\right),
$$

more relevant to dynamical stability, the typical spacing is 10-30 (Fang and Margot 2013). At the lower end of this distribution, the systems flirt with instability (Deck et al. 2012, $\mathrm{Pu}$ and $\mathrm{Wu}$ 2015). A few percent of the Kepler systems are in (or near) mean-motion resonances, suggesting that the orbits have been sculpted by planet-disk gravitational interactions. These systems offer the gift of transit-timing variations (Agol \& Fabrycky, this volume), the observable manifestations of planetplanet gravitational interactions that sometimes allow for measurements of planetary masses as well as orbital eccentricities and inclinations. Such studies and some other lines of evidence show that the compact multiple-planet systems tend to have orbits that are nearly circular (Hadden and Lithwick|2014, Xie et al.|2016, Van Eylen and Albrecht 2015) and coplanar (Fabrycky et al. 2014).

Radius gap The radius distribution of planets with periods shorter than 100 days shows a dip in occurrence between 1.5 and $2 R_{\oplus}$ (Fulton et al. 2017, Van Eylen et al. 2017, see Figure 5p. Such a feature had been anticipated based on theoretical calculations of the photo-evaporation of the atmospheres of low-mass planets by the intense radiation from the host star (Owen and Wu 2013, Lopez and Fortney 2013). Thus, the radius gap or "evaporation valley" seems to be a precious example in exoplanetary science of a prediction fulfilled, with many implications for the structures and atmospheres of close-orbiting planets (Owen and Wu 2017).

Hot Neptunes As mentioned above, $d n / d \log P$ changes from a rising function for $P \lesssim 10$ days to a more constant value for $P=10-100$ days. The critical period separating these regimes is longer for larger planets. The effect is to create a diagonal boundary in the space of $\log R$ and $\log P$, above which the occurrence is very low (see Fig. 44. The same phenomenon is seen in Doppler data (Mazeh et al. 2016). This "hot Neptune desert" may be another consequence of atmospheric erosion. Interestingly those few hot Neptunes that do exist are strongly associated with metal-rich stars (Dong et al. 2017, Petigura et al. 2018), making them similar to giant planets and unlike smaller planets. The hot Neptunes are also similar to hot Jupiters in that they tend not to have planetary companions in closely-spaced coplanar orbits (Dong et al. 2017). All this suggests the hot Neptunes and close-in giant planets originate in similar circumstances, possibly from some type of dynamical instability. 


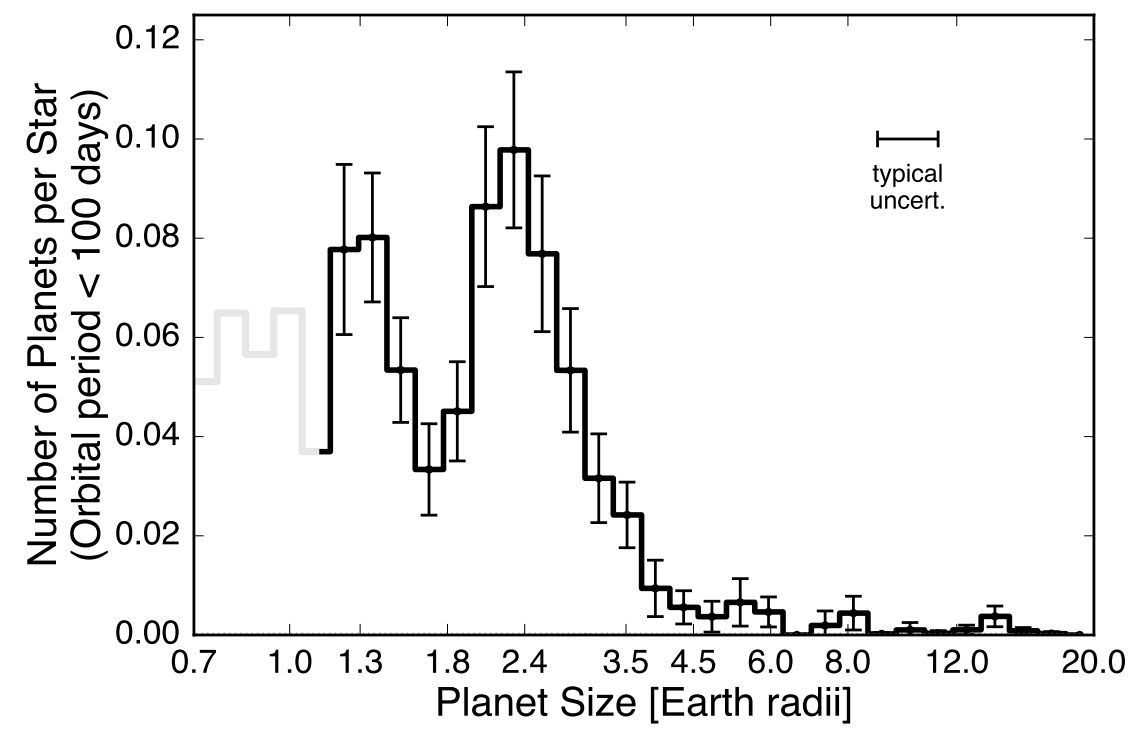

Fig. 5 From Fulton et al. 2017). Occurrence as a function of radius based on Kepler data, for orbital periods shorter than 100 days. The dip in the occurrence rate density between $1.5-2 R_{\oplus}$ has been attributed to the erosion of planetary atmospheres by high-energy radiation from the star.

Earth-like planets A goal with broad appeal is measuring the occurrence rate of Earth-sized planets orbiting Sun-like stars within the "habitable zone", the range of distances within which a rocky planet could plausibly have oceans of liquid water. The Kepler mission provided the best-ever data for this purpose. However, even Kepler was barely sensitive to such planets. The number of detections was of order 10 , depending on the definitions of "Earth-sized", "Sun-like" and "habitable zone". The desired quantity can be understood as an integral

$$
\eta_{\oplus} \equiv \int_{R_{\min }}^{R_{\max }} \int_{S_{\min }}^{S_{\max }} \frac{\partial^{2} n}{\partial \log S \partial \log R} d \log S d \log R
$$

where $S$ is the bolometric flux the planet receives from the star. The integration limits are chosen to select planets likely to have a solid surface with a temperature and pressure allowing for liquid water. These limits depend on assumptions about the structure and atmosphere of the planet and the spectrum of the star (see, e.g., Kasting et al. 2014).

Even if we set aside the problem of setting the integration limits, the measurement of

$$
\left.\Gamma_{\oplus} \equiv \frac{\partial^{2} n}{\partial \log P \partial \log R}\right|_{P=1 \mathrm{yr}, R=R_{\oplus}}
$$


has proven difficult and may require extrapolation from measurements of larger planets at shorter periods. The Kepler team has published a series of papers reporting steady advancement in the efficiency of detection, elimination of false positives, and understanding of instrumental artifacts. The most recent effort to determine $\Gamma_{\oplus}$ found the data to be compatible with values ranging from 0.04 to 11.5 (see Figure 6). Since then the Kepler team and other groups have clarified the properties of the stars that were searched (Petigura et al. 2017, De Cat et al. 2015), and the most recent installments by Twicken et al. (2016) and Thompson et al. (2017) quantified the sensitivity of the algorithms for planet detection and validation. These developments have brought us right to the threshold of an accurate occurrence rate for Earth-like planets.

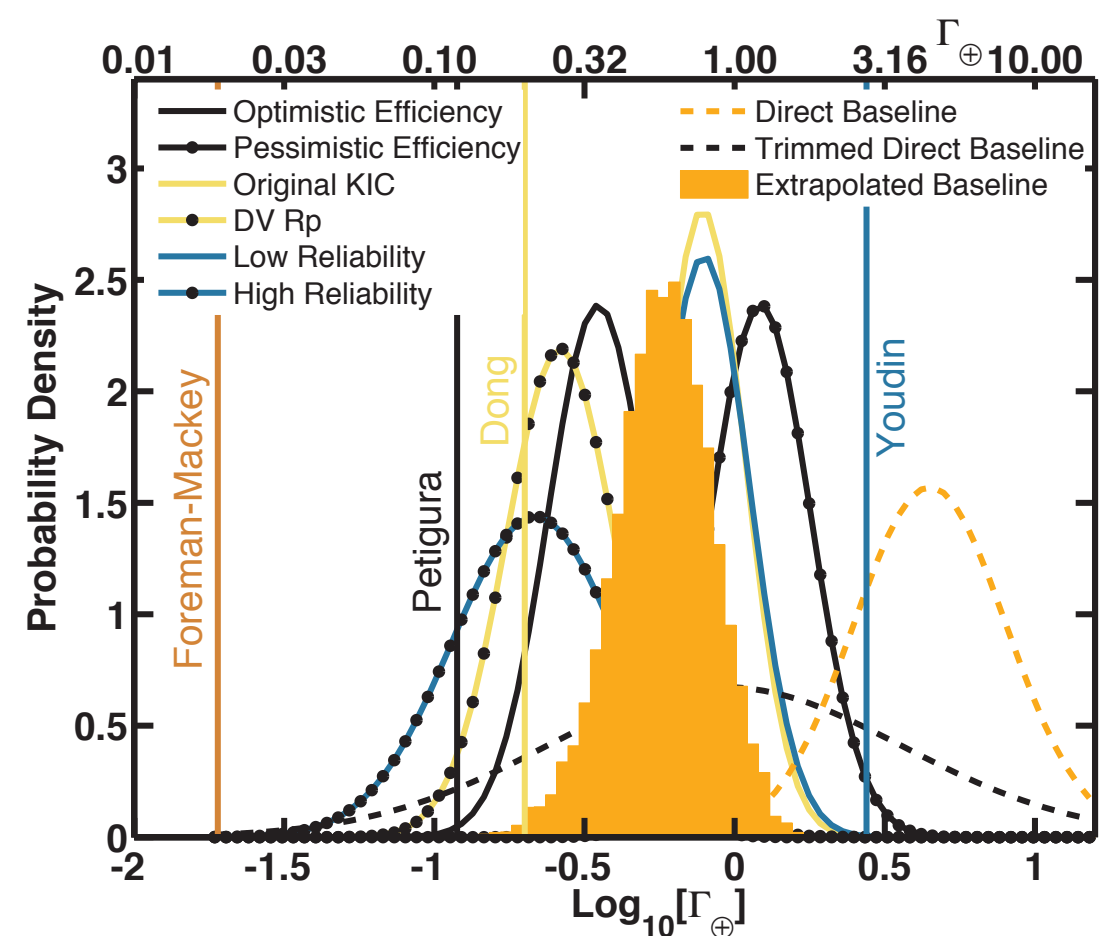

Fig. 6 From Burke et al. (2015). Estimates for $\Gamma_{\oplus}$ based on Kepler data. The orange histogram is the posterior probability distribution considering only the uncertainties from counting statistics and extrapolation. The other curves illustrate the effects of some systematic errors: uncertainty in the detection efficiency, orbital eccentricities, stellar parameters, and reliability of weak planet candidates. These systematic effects led to a range in $\Gamma_{\oplus}$ spanning an order of magnitude. The vertical lines show other estimates of $\Gamma_{\oplus}$ by Foreman-Mackey et al. (2014); Petigura et al. (2013); Dong and Zhu (2013) and Youdin (2011). 


\section{Other types of stars}

Almost all the preceding results pertain to main-sequence stars with masses between 0.5 and $1.2 M_{\odot}$, i.e., spectral types from K to late F. Stars with masses between 0.1 and $0.5 M_{\odot}$, the $\mathrm{M}$ dwarfs, are not as thoroughly explored, especially near the low end of the mass range. However these stars are very attractive for planet surveys because their small masses and sizes lead to larger Doppler and transit signals, and because planets in the habitable zone have conveniently short orbital periods.

Giant planets are relatively rare around $\mathrm{M}$ dwarfs, at least for periods shorter than a few years. Cumming et al. (2008) showed that if planet occurrence is modeled by the functional form of Equation (5), then planets with masses exceeding $0.4 M_{\text {Jup }}$ and periods shorter than 5.5 years are 3-10 times less common around M dwarfs than around FGK dwarfs. Similar results were obtained by Bonfils et al. (2013).

On the other hand, for smaller planets over the same range of periods, M dwarf occurrence rates exceed those of FGK dwarfs by a factor of 2-3 (Howard et al. 2012; Mulders et al. 2015). This result is based on Kepler data, which remains our best source of information on this topic despite the fact that only a few percent of the Kepler target stars were M dwarfs. Comprehensive analyses have been performed by Dressing and Charbonneau (2015) and Gaidos et al. (2016). Their results differ in detail but agree that on average, $\mathrm{M}$ dwarfs have about two planets per star with a radius in between those of Earth and Neptune, and an orbital period shorter than 100 days (see Figure 4). One implication of this high occurrence rate is that the nearest habitable-zone planets are almost surely around M dwarfs. Indeed, Doppler surveys have turned up two candidates for "temperate" Earth-mass planets around M dwarfs within just a few parsecs: Proxima Cen (1.3 pc, Anglada-Escudé et al. 2016) and Ross 128 (also known as Proxima Vir; 3.4 pc, Bonfils et al. 2017).

Some other comparisons with FGK dwarfs have been made. Among the similarities are that $\mathrm{M}$ dwarfs often have compact systems of multiple planets (Muirhead et al. 2015; Gaidos et al. 2016, Ballard and Johnson 2016), and that high metallicity is associated with giant planet occurrence (Johnson et al. 2010, Neves et al. 2013). It remains unclear whether or not the occurrence of smaller planets is associated with high metallicity for M dwarfs (see, e.g., Gaidos and Mann 2014). There is also evidence that the planet population around M dwarfs exhibit both the "evaporation valley" between 1.5-2 $R_{\oplus}$ and the "hot Neptune desert" (Hirano et al. 2017).

Beyond the scope of this review, but nevertheless fascinating, are the occurrence rates that have been measured in Doppler and transit surveys of other types of stars: evolved stars (Johnson et al. 2010; Reffert et al. 2015), stars in open clusters (Mann et al.2017) and globular clusters (Gilliland et al. 2000; Masuda and Winn|2017), binary stars (Armstrong et al. 2014), brown dwarfs (He et al.2017), and white dwarfs (Fulton et al. 2014, van Sluijs and Van Eylen 2018). 


\section{Future Prospects}

The data from recent surveys offer opportunities for progress for at least another few years. The ongoing struggle to measure the occurrence rate of Earth-like planets has already been described. Another undeveloped area is the determination of joint and conditional probabilities; for example, given a planet with radius $R_{1}$ and period $P_{1}$, what is the chance of finding another planet around the same star with radius $R_{2}$ and period $P_{2}$ ? Conditional rates, or the relative occurrence of different types of systems, may be more useful than overall occurrence rates for testing planet formation theories. Only a few cases have been studied, such as the mutual radius distribution of neighboring planets (Ciardi et al. 2013; Weiss et al. 2018), and the probability for giant planets to have wider-orbiting companions (Huang et al. 2016; Bryan et al. 2016; Schlaufman and Winn 2016).

New data are also forthcoming. The stellar parallaxes soon to be available from the ESA Gaia mission (Gaia Collaboration et al. 2016) will clarify the properties of all the Kepler stars as well as the targets of future surveys. Among these future surveys is the Transiting Exoplanet Survey Satellite (TESS), scheduled for launch in 2018 (Ricker et al. 2015). This mission was not designed to measure planet occurrence rates, but rather to pluck low-hanging fruit: short-period transiting planets around bright stars. Less well appreciated is that TESS may be superior to Kepler for measuring the occurrence of planets larger than Neptune with periods shorter than 10 days. When TESS was conceived it was expected that limitations in data storage and transmission would restrict the search to $\approx 10^{5}$ pre-selected stars, as was the case with Kepler. Later it became clear that entire TESS images could be stored and transmitted with 30 -minute time sampling. As a result, although $\approx 10^{5}$ Sun-like stars will still be selected for finer time sampling, it should be possible to search millions of stars for large and short-period planets. This includes hot and massive stars, for which comparatively little is known. TESS should excel at finding rare, large-amplitude, short-period photometric phenomena of all kinds.

For smaller planets around Sun-like stars, it will be difficult to achieve an orderof-magnitude improvement over the existing data. There is more room for advances in the study of low-mass stars, using new Doppler spectrographs operating at far-red and infrared wavelengths, and ground-based transit surveys focusing exclusively on low-mass stars. Particularly encouraging was the discovery of TRAPPIST-1, a system of seven Earth-sized planets orbiting an "ultra-cool dwarf" that barely qualifies as a star (Gillon et al. 2017). This system was found after searching $\approx 50$ similar objects with a detection efficiency of around 60\% (Burdanov et al., this volume; M. Gillon, private communication), and the transit probability for the innermost planet is $5 \%$. This suggests the occurrence of such systems is approximately $(50 \cdot 0.6 \cdot 0.05)^{-1}=0.7$. Thus, while TRAPPIST-1 seems extraordinary, it may represent a typical outcome of planet formation around ultra-cool dwarfs.

In the decades to come, the domains of all the planet detection techniques including direct imaging, gravitational microlensing, and astrometry - will begin overlapping. Some efforts have already been made to determine occurrence rate densities based on data from very different techniques (see, e.g. Montet et al. 2014; 
Clanton and Gaudi 2016). We can look forward to a more holistic view of the occurrence of planets around other stars, barring any civilization-ending cataclysm.

Acknowledgements The author is grateful to Hans Deeg and Natalie Batalha for the invitation to write this review and for their editorial guidance; Bill Borucki, Luke Bouma, Fei Dai, Courtney Dressing, Kento Masuda, Erik Petigura, and Saul Rappaport, for comments on the manuscript; and Chris Burke, Courtney Dressing, B.J. Fulton, Erik Petigura, and Alexandre Santerne for allowing the use of the data and figures from their papers.

\section{References}

Anglada-Escudé G, Amado PJ, Barnes J et al. (2016) A terrestrial planet candidate in a temperate orbit around Proxima Centauri. Nature536:437-440

Armstrong DJ, Osborn HP, Brown DJA et al. (2014) On the abundance of circumbinary planets. MNRAS444:1873-1883

Ballard S Johnson JA (2016) The Kepler Dichotomy among the M Dwarfs: Half of Systems Contain Five or More Coplanar Planets. ApJ816:66

Bonfils X, Delfosse X, Udry S et al. (2013) The HARPS search for southern extra-solar planets. XXXI. The M-dwarf sample. A\&A549:A109

Bonfils X, Astudillo-Defru N, Díaz R et al. (2017) A temperate exo-Earth around a quiet M dwarf at 3.4 parsecs. ArXiv e-prints

Borucki WJ (2016) KEPLER Mission: development and overview. Reports on Progress in Physics 79(3):036901

Bryan ML, Knutson HA, Howard AW et al. (2016) Statistics of Long Period Gas Giant Planets in Known Planetary Systems. ApJ821:89

Buchhave LA, Latham DW, Johansen A et al. (2012) An abundance of small exoplanets around stars with a wide range of metallicities. Nature 486:375-377

Burke CJ, Christiansen JL, Mullally F et al. (2015) Terrestrial Planet Occurrence Rates for the Kepler GK Dwarf Sample. ApJ809:8

Chiang E Laughlin G (2013) The minimum-mass extrasolar nebula: in situ formation of close-in super-Earths. MNRAS431:3444-3455

Ciardi DR, Fabrycky DC, Ford EB et al. (2013) On the Relative Sizes of Planets within Kepler Multiple-candidate Systems. ApJ763:41

Clanton C Gaudi BS (2016) Synthesizing Exoplanet Demographics: A Single Population of Longperiod Planetary Companions to M Dwarfs Consistent with Microlensing, Radial Velocity, and Direct Imaging Surveys. ApJ819:125

Cumming A (2004) Detectability of extrasolar planets in radial velocity surveys. MNRAS354:1165-1176

Cumming A, Butler RP, Marcy GW et al. (2008) The Keck Planet Search: Detectability and the Minimum Mass and Orbital Period Distribution of Extrasolar Planets. PASP120:531

De Cat P, Fu JN, Ren AB et al. (2015) Lamost Observations in the Kepler Field. I. Database of Low-resolution Spectra. ApJS220:19

Deck KM, Holman MJ, Agol E et al. (2012) Rapid Dynamical Chaos in an Exoplanetary System. ApJ755:L21

Dong S Zhu Z (2013) Fast Rise of "Neptune-size" Planets (4-8 $\mathrm{R}_{\oplus}$ ) from P = 10 to 250 DaysStatistics of Kepler Planet Candidates up to 0.75 AU. ApJ778:53

Dong S, Xie JW, Zhou JL, Zheng Z Luo A (2017) LAMOST telescope reveals that Neptunian cousins of hot Jupiters are mostly single offspring of stars that are rich in heavy elements. ArXiv e-prints 
Dressing CD Charbonneau D (2015) The Occurrence of Potentially Habitable Planets Orbiting M Dwarfs Estimated from the Full Kepler Dataset and an Empirical Measurement of the Detection Sensitivity. ApJ807:45

Fabrycky DC, Lissauer JJ, Ragozzine D et al. (2014) Architecture of Kepler's Multi-transiting Systems. II. New Investigations with Twice as Many Candidates. ApJ790:146

Fang J Margot JL (2013) Are Planetary Systems Filled to Capacity? A Study Based on Kepler Results. ApJ767:115

Feynman RP (1963) Feynman lectures on physics - Volume 1

Figueira P, Marmier M, Boué G et al. (2012) Comparing HARPS and Kepler surveys. The alignment of multiple-planet systems. A\&A541:A139

Fischer DA Valenti J (2005) The Planet-Metallicity Correlation. ApJ622:1102-1117

Foreman-Mackey D, Hogg DW Morton TD (2014) Exoplanet Population Inference and the Abundance of Earth Analogs from Noisy, Incomplete Catalogs. ApJ 795:64

Foreman-Mackey D, Morton TD, Hogg DW, Agol E Schölkopf B (2016) The Population of Longperiod Transiting Exoplanets. AJ152:206

Fressin F, Torres G, Charbonneau D et al. (2013) The False Positive Rate of Kepler and the Occurrence of Planets. ApJ766:81

Fulton BJ, Tonry JL, Flewelling H et al. (2014) A Search for Planetary Eclipses of White Dwarfs in the Pan-STARRS1 Medium-deep Fields. ApJ796:114

Fulton BJ, Petigura EA, Howard AW et al. (2017) The California-Kepler Survey. III. A Gap in the Radius Distribution of Small Planets. AJ154:109

Gaia Collaboration, Prusti T, de Bruijne JHJ et al. (2016) The Gaia mission. A\&A595:A1

Gaidos E Mann AW (2014) M Dwarf Metallicities and Giant Planet Occurrence: Ironing Out Uncertainties and Systematics. ApJ791:54

Gaidos E, Mann AW, Kraus AL Ireland M (2016) They are small worlds after all: revised properties of Kepler M dwarf stars and their planets. MNRAS457:2877-2899

Gilliland RL, Brown TM, Guhathakurta P et al. (2000) A Lack of Planets in 47 Tucanae from a Hubble Space Telescope Search. ApJ545:L47-L51

Gillon M, Triaud AHMJ, Demory BO et al. (2017) Seven temperate terrestrial planets around the nearby ultracool dwarf star TRAPPIST-1. Nature542:456-460

Grether D Lineweaver CH (2006) How Dry is the Brown Dwarf Desert? Quantifying the Relative Number of Planets, Brown Dwarfs, and Stellar Companions around Nearby Sun-like Stars. ApJ640:1051-1062

Guo X, Johnson JA, Mann AW et al. (2017) The Metallicity Distribution and Hot Jupiter Rate of the Kepler Field: Hectochelle High-resolution Spectroscopy for 776 Kepler Target Stars. ApJ838:25

Hadden S Lithwick Y (2014) Densities and Eccentricities of 139 Kepler Planets from Transit Time Variations. ApJ787:80

Hansen BMS Murray N (2012) Migration Then Assembly: Formation of Neptune-mass Planets inside 1 AU. ApJ751:158

He MY, Triaud AHMJ Gillon M (2017) First limits on the occurrence rate of short-period planets orbiting brown dwarfs. MNRAS464:2687-2697

Hirano T, Dai F, Gandolfi D et al. (2017) Planetary Systems around Low-mass Stars Unveiled by K2. ArXiv e-prints

Howard AW, Marcy GW, Johnson JA et al. (2010) The Occurrence and Mass Distribution of Closein Super-Earths, Neptunes, and Jupiters. Science 330:653

Howard AW, Marcy GW, Bryson ST et al. (2012) Planet Occurrence within 0.25 AU of Solar-type Stars from Kepler. ApJS201:15

Huang C, Wu Y Triaud AHMJ (2016) Warm Jupiters Are Less Lonely than Hot Jupiters: Close Neighbors. ApJ825:98

Ida S Lin DNC (2008) Toward a Deterministic Model of Planetary Formation. V. Accumulation Near the Ice Line and Super-Earths. ApJ685:584-595

Johnson JA, Howard AW, Bowler BP et al. (2010) Retired A Stars and Their Companions. IV. Seven Jovian Exoplanets from Keck Observatory. PASP122:701 
Kasting JF, Kopparapu R, Ramirez RM Harman CE (2014) Remote life-detection criteria, habitable zone boundaries, and the frequency of Earth-like planets around $\mathrm{M}$ and late $\mathrm{K}$ stars. Proceedings of the National Academy of Science 111:12,641-12,646

Lecar M, Podolak M, Sasselov D Chiang E (2006) On the Location of the Snow Line in a Protoplanetary Disk. ApJ640:1115-1118

Lissauer JJ, Ragozzine D, Fabrycky DC et al. (2011) Architecture and Dynamics of Kepler's Candidate Multiple Transiting Planet Systems. ApJS197:8

Lopez ED Fortney JJ (2013) The Role of Core Mass in Controlling Evaporation: The Kepler Radius Distribution and the Kepler-36 Density Dichotomy. ApJ776:2

Lovis C Fischer D (2010) Radial Velocity Techniques for Exoplanets, pp 27-53

Mann AW, Gaidos E, Vanderburg A et al. (2017) Zodiacal Exoplanets in Time (ZEIT). IV. Seven Transiting Planets in the Praesepe Cluster. AJ153:64

Masuda K Winn JN (2017) Reassessment of the Null Result of the HST Search for Planets in 47 Tucanae. AJ153:187

Mayor M, Marmier M, Lovis C et al. (2011) The HARPS search for southern extra-solar planets XXXIV. Occurrence, mass distribution and orbital properties of super-Earths and Neptunemass planets. arxiv: 11092497

Mazeh T, Holczer T Faigler S (2016) Dearth of short-period Neptunian exoplanets: A desert in period-mass and period-radius planes. A\&A589:A75

Montet BT, Crepp JR, Johnson JA, Howard AW Marcy GW (2014) The TRENDS High-contrast Imaging Survey. IV. The Occurrence Rate of Giant Planets around M Dwarfs. ApJ781:28

Muirhead PS, Mann AW, Vanderburg A et al. (2015) Kepler-445, Kepler-446 and the Occurrence of Compact Multiples Orbiting Mid-M Dwarf Stars. ApJ801:18

Mulders GD, Pascucci I Apai D (2015) An Increase in the Mass of Planetary Systems around Lower-mass Stars. ApJ814:130

Mulders GD, Pascucci I, Apai D, Frasca A Molenda-Zakowicz J (2016) A Super-solar Metallicity for Stars with Hot Rocky Exoplanets. AJ152:187

Neves V, Bonfils X, Santos NC et al. (2013) Metallicity of M dwarfs. III. Planet-metallicity and planet-stellar mass correlations of the HARPS GTO M dwarf sample. A\&A551:A36

Owen JE Wu Y (2013) Kepler Planets: A Tale of Evaporation. ApJ775:105

Owen JE Wu Y (2017) The Evaporation Valley in the Kepler Planets. ApJ847:29

Pepper J, Gould A Depoy DL (2003) Using All-Sky Surveys to Find Planetary Transits. Acta Astron53:213-228

Petigura EA, Howard AW Marcy GW (2013) Prevalence of Earth-size planets orbiting Sun-like stars. Proceedings of the National Academy of Science 110:19,273-19,278

Petigura EA, Howard AW, Marcy GW et al. (2017) The California-Kepler Survey. I. Highresolution Spectroscopy of 1305 Stars Hosting Kepler Transiting Planets. AJ154:107

Petigura EA, Marcy GW, Winn JN et al. (2018) The California-Kepler Survey. IV. Metal-rich Stars Host a Greater Diversity of Planets. AJ155:89

Pollack JB, Hubickyj O, Bodenheimer P et al. (1996) Formation of the Giant Planets by Concurrent Accretion of Solids and Gas. Icarus 124:62-85

Pu B Wu Y (2015) Spacing of Kepler Planets: Sculpting by Dynamical Instability. ApJ807:44

Reffert S, Bergmann C, Quirrenbach A, Trifonov T Künstler A (2015) Precise radial velocities of giant stars. VII. Occurrence rate of giant extrasolar planets as a function of mass and metallicity. A\&A574:A116

Ricker GR, Winn JN, Vanderspek R et al. (2015) Transiting Exoplanet Survey Satellite (TESS). Journal of Astronomical Telescopes, Instruments, and Systems 1(1):014003

Sahlmann J, Ségransan D, Queloz D et al. (2011) Search for brown-dwarf companions of stars. A\&A525:A95

Sanchis-Ojeda R, Rappaport S, Winn JN et al. (2014) A Study of the Shortest-period Planets Found with Kepler. ApJ787:47

Santerne A, Moutou C, Tsantaki M et al. (2016) SOPHIE velocimetry of Kepler transit candidates. XVII. The physical properties of giant exoplanets within 400 days of period. A\&A587:A64 
Santos NC, Israelian G, Mayor M, Rebolo R Udry S (2003) Statistical properties of exoplanets. II. Metallicity, orbital parameters, and space velocities. A\&A398:363-376

Santos NC, Adibekyan V, Figueira P et al. (2017) Observational evidence for two distinct giant planet populations. A\&A603:A30

Schlaufman KC (2018) Evidence of an Upper Bound on the Masses of Planets and Its Implications for Giant Planet Formation. ApJ853:37

Schlaufman KC Winn JN (2016) The Occurrence of Additional Giant Planets Inside the Water-Ice Line in Systems with Hot Jupiters: Evidence Against High-Eccentricity Migration. ApJ825:62

Shallue CJ Vanderburg A (2018) Identifying Exoplanets with Deep Learning: A Five-planet Resonant Chain around Kepler-80 and an Eighth Planet around Kepler-90. AJ155:94

Tabachnik S Tremaine S (2002) Maximum-likelihood method for estimating the mass and period distributions of extrasolar planets. MNRAS335:151-158

Thompson SE, Coughlin JL, Hoffman K et al. (2017) Planetary Candidates Observed by Kepler. VIII. A Fully Automated Catalog With Measured Completeness and Reliability Based on Data Release 25. ArXiv e-prints

Tremaine S Dong S (2012) The Statistics of Multi-planet Systems. AJ143:94

Triaud AHMJ, Martin DV, Ségransan D et al. (2017) The EBLM Project. IV. Spectroscopic orbits of over 100 eclipsing M dwarfs masquerading as transiting hot Jupiters. A\&A608:A129

Twicken JD, Jenkins JM, Seader SE et al. (2016) Detection of Potential Transit Signals in 17 Quarters of Kepler Data: Results of the Final Kepler Mission Transiting Planet Search (DR25). AJ152:158

Udry S Santos NC (2007) Statistical Properties of Exoplanets. ARA\&A45:397-439

Van Eylen V Albrecht S (2015) Eccentricity from Transit Photometry: Small Planets in Kepler Multi-planet Systems Have Low Eccentricities. ApJ808:126

Van Eylen V, Agentoft C, Lundkvist MS et al. (2017) An asteroseismic view of the radius valley: stripped cores, not born rocky. ArXiv e-prints

van Sluijs L Van Eylen V (2018) The occurrence of planets and other substellar bodies around white dwarfs using K2. MNRAS474:4603-4611

Wang J, Fischer DA, Horch EP Huang X (2015) On the Occurrence Rate of Hot Jupiters in Different Stellar Environments. ApJ799:229

Weiss LM, Marcy GW, Petigura EA et al. (2018) The California-Kepler Survey. V. Peas in a Pod: Planets in a Kepler Multi-planet System Are Similar in Size and Regularly Spaced. AJ155:48

Wilson RF, Teske J, Majewski SR et al. (2018) Elemental Abundances of Kepler Objects of Interest in APOGEE. I. Two Distinct Orbital Period Regimes Inferred from Host Star Iron Abundances. AJ155:68

Winn JN (2010) Exoplanet Transits and Occultations, University of Arizona Press, pp 55-77

Winn JN Fabrycky DC (2015) The Occurrence and Architecture of Exoplanetary Systems. ARA\&A53:409-447

Wittenmyer RA, Butler RP, Tinney CG et al. (2016) The Anglo-Australian Planet Search XXIV: The Frequency of Jupiter Analogs. ApJ819:28

Wolfgang A Laughlin G (2012) The Effect of Population-wide Mass-to-radius Relationships on the Interpretation of Kepler and HARPS Super-Earth Occurrence Rates. ApJ750:148

Wright JT, Veras D, Ford EB et al. (2011) The California Planet Survey. III. A Possible 2:1 Resonance in the Exoplanetary Triple System HD 37124. ApJ730:93

Wright JT, Marcy GW, Howard AW et al. (2012) The Frequency of Hot Jupiters Orbiting nearby Solar-type Stars. ApJ753:160

Xie JW, Dong S, Zhu Z et al. (2016) Exoplanet orbital eccentricities derived from LAMOST-Kepler analysis. Proceedings of the National Academy of Science 113:11,431-11,435

Youdin AN (2011) The Exoplanet Census: A General Method Applied to Kepler. ApJ742:38 